\title{
Cytoplasmic versus nuclear THR alpha expression determines survival of ovarian cancer patients
}

\author{
Nina Ditsch ${ }^{1,5}$. Sabine Heublein ${ }^{1,2}$. Udo Jeschke ${ }^{1,5} \cdot$ Cornelia Sattler $^{1} \cdot$ Christina Kuhn $^{1}$ - Anna Hester ${ }^{1}$. \\ Bastian Czogalla ${ }^{1} \cdot$ Fabian Trillsch $^{1} \cdot$ Sven Mahner ${ }^{1} \cdot$ Jutta Engel $^{3} \cdot$ Doris Mayr $^{4} \cdot$ Elisa Schmoeckel $^{4} \mathbb{C}$
}

Received: 2 February 2020 / Accepted: 1 May 2020 / Published online: 12 June 2020

(c) The Author(s) 2020

\begin{abstract}
Purpose Thyroid hormone receptors (THR) have manifold functions and are involved in the carcinogenesis of several tumor types. Within this study, we aimed to investigate the expression pattern (nuclear versus cytoplasmic) of the THR alpha and its impact on patients survival in ovarian cancer $(\mathrm{OvCa})$.

Methods The presence of the thyroid hormone receptors THR $\alpha$, THR $\alpha 1$ and -2 was investigated in 156 ovarian cancer samples using immunohistochemistry (IHC) using semi-quantitative immunoreactivity (IR) scores and correlated with clinical, pathological data, subtype of ovarian cancer, clinical data, staining of 20 already described OvCa marker proteins and overall survival (OS).

Results Among all subtypes of OvCa, clear cell carcinomas showed the highest THR $\alpha$ expression. Furthermore, nuclear THR $\alpha$ was associated with a reduced survival in this subtype. However, nuclear expressed THR $\alpha 1$ turned out to be a positive prognosticator for all subtypes of OvCa patients. Nuclear THR $\alpha 2$ is a positive prognosticator for OvCa patients of the serous subtype. In contrast, cytoplasmic expression THR $\alpha 2$ was associated with a reduced OS in all subtypes of OvCa patients; while, cytoplasmic expression of THR $\alpha 1$ is associated with reduced OS in mucinous OvCa patients only. In addition, THR $\alpha$ expression correlates with gonadotropin receptors, steroid hormone receptors, TA-MUC1 and glycodelin.

Conclusion Depending on nuclear or cytoplasmic expression, our study shows that THR $\alpha$ and its isoforms 1 and 2 provide different prognostic information for ovarian cancer patients. Further investigations should analyze if THRs may represent new endocrine targets for the treatment of ovarian cancer.
\end{abstract}

Keywords Thyroid hormone receptor $\cdot$ Ovarian cancer $\cdot$ Overall survival $\cdot$ Nuclear versus cytoplasmic

\section{Background}

Thyroid-stimulating hormone (TSH) regulates thyroid function by binding to its receptor (thyroid hormone receptorTHR) expressed at the surface of thyroid cells. Recently, it has been demonstrated that THR is abundantly expressed in several tissues apart from the thyroid, among them the normal ovarian surface epithelium. The hormone dependency

Nina Ditsch and Sabine Heublein contributed equally to the study.

Electronic supplementary material The online version of this article (https://doi.org/10.1007/s00432-020-03241-7) contains supplementary material, which is available to authorized users.

Udo Jeschke

udo.jeschke@med.unimuenchen.de

Extended author information available on the last page of the article of the ovaries and the functional similarity of THRs and estrogen- (ER) and progesterone receptors (PR; both act in the nucleus as transcription factors) lead to the hypothesis that THRs may be a prognostic marker in ovarian cancer patients as demonstrated recently for breast cancer patients (Li et al. 2002; Rasmusson et al. 1987; Turken et al. 2003; Ditsch et al. 2013).

The nuclear receptors of thyroid hormones regulate the expression of specific cellular genes by interacting with distinct DNA sequences. They are ligand-activated transcription factors, which regulate the transcription of target genes. THRs are encoded by two genes-THR alpha and beta-located on human chromosomes 17 and 3 (Silva et al. 2002). They have three major isoforms: THR $\alpha 1, T H R \alpha 2$ and THR $\beta 1$ (Ling et al. 2010) with high homology in amino acid composition. The most diversified region between THR $\alpha$ and THR $\beta$ is located in the $\mathrm{N}$-terminal area, related to their 
trans-activation activity (Lazar 1993). Recent studies discovered by oligonucleotide microarray transcriptional profiling that THR $\alpha$ and THR $\beta$ mRNAs are among the most strongly expressed nuclear hormone receptor genes in cultured human ovarian surface epithelial (OSE) cells (Rae et al. 2004). The presence of THR $\alpha 1$, THR $\alpha 2$, and THR $\beta 1$ transcripts in cultured OSE cells is confirmed and the presence of THR $\alpha$ and THR $\beta$ proteins in the OSE cell layer has been demonstrated. Although, THR $\alpha$ and $\beta$ isoforms are encoded by separate genes, differential promoter usage gives rise to two different THR $\alpha$ receptors, THR $\alpha 1$ and THR $\alpha 2$ (Zhang and Lazar 2000). Unlike THR $\alpha 1$ and THR $\beta 1$, which are conventional ligand-activated receptors, THR $\alpha 2$ is a ligandindependent negative regulator of active THRs. Thus, the presence of different THR isoforms, in conjunction with the potential for pre-receptor metabolism of thyroid hormones through expression of activating and inactivating deiodinase enzymes, strengthens the likelihood that the OSE is a physiologically important thyroid hormone target tissue (Rae et al. 2004).

Ovulation is a recurrent inflammatory reaction causing regular and frequent local injury to the ovarian surface during follicular rupture (Espey 1994; Rae and Hillier 2005). Ovarian cancer develops when a mutation or genetic change - spossibly caused by repeat episodes of inflammation-associated DNA damage (Murdoch 1998; Murdoch et al. 1999; Beachy et al. 2004)—occurs in the cells on the surface of the ovaries or in the fallopian tubes and leads to uncontrolled cell growth that may often metastasize (Rasool et al. 2014). Suppression of ovulation by e.g. pregnancy, breast feeding, or oral contraception reduces the risk of ovarian cancer, whereas diseases such as endometriosis, ovarian cysts, and hyperthyroidism are associated with increased risk (Ness and Cottreau 1999; Ness et al. 2000).

Ovarian cancer consists of four histopathological subtypes, represents the fourth most frequent type of cancer among females, and is the leading cause of death from gynecological cancer in the western world. Besides the histopathological subtype, grading, clinical staging and the amount of residual tumor, a number of several putative prognostic markers had been suggested for monitoring this disease (Ditsch et al. 2013). As ovarian cancer is also a thyroid hormone-dependent neoplasm (Shinderman-Maman et al. 2016), T3 has been shown to directly exert inflammatory effects on ovarian surface epithelial cell function in vitro and activate expression of genes associated with inflammation (Cohen et al. 2014; Rae et al. 2007). Studies also indicate that $\mathrm{T} 3$ increases the expression of ER $\alpha$, which strongly associates with the development of epithelial ovarian cancer, which may explain the epidemiological linkage between hyperthyroidism and ovarian cancer (Rae et al. 2007).

The current study examines possible alterations of THR expression in ovarian carcinomas and its implication in ovarian cancer survival. Little is known about the context of thyroid function in ovarian carcinogenesis and the role of THR expression outside the thyroid is not completely understood. From our knowledge of therapy modalities, anti-hormonal therapy like tamoxifen, which unfold its effect via steroid hormone receptors, can be affective in ER-positive ovarian cancers. First in this field, our examinations focuses on the prognostic impact of thyroid hormone receptors of the alpha subtype (general alpha, alpha-1 and alpha-2, respectively) on pathological different ovarian cancer tissues.

\section{Methods}

\section{Tissue samples}

Tissue samples were obtained from 156 patients undergoing gynecological surgery for epithelial ovarian cancer (EOC) at the Department of Obstetrics and Gynaecology of the Ludwig-Maximilians-University Munich. The clinico-pathological parameters are shown in Table 1. Experienced gynaecologic pathologists performed histopathological staining and evaluation according to the criteria of the International Federation of Gynaecologists and Obstetricians (FIGO) and the World Health Organization (WHO). Full slides were used for immuohistochemical stainings. EOC specimens were available in different histological subtypes: serous $(n=110)$ thereof 84 high-grade and 26 low-grade cases, clear cell $(n=12)$, endometrioid $(n=21)$, mucinous $(n=13)$. Patients with ovarian low malignant potential tumors (e.g., Borderline tumors) were excluded from the study and no patients had neo-adjuvant chemotherapy. Patient's clinical data were available from patient charts, aftercare files and tumor registry database information. The main outcomes assessed were disease recurrence and patient survival. For
Table 1 Clinico-pathological parameters of the study group $(n=156)$

\begin{tabular}{llllllll}
\hline Histological subtype & & \multicolumn{2}{l}{ FIGO stage } & \multicolumn{2}{l}{ Nodal status } & \multicolumn{2}{l}{ Age (years) } \\
\hline High-grade serous & $84(54 \%)$ & I & $35(23 \%)$ & N0 & $56(36 \%)$ & mean & 62 \\
Low-grade serous & $26(17 \%)$ & II & $10(6 \%)$ & N1 & $54(35 \%)$ & min & 33 \\
Endometrioid & $21(13 \%)$ & III & $107(69 \%)$ & NX & $46(29 \%)$ & max & 88 \\
Mucinous & $13(8 \%)$ & IV & $4(2 \%)$ & & & & \\
Clear cell & $12(8 \%)$ & & & & & \\
\hline
\end{tabular}


survival analysis, survival time was defined as the time between the date of primary ovarian cancer diagnosis and the date of death.

\section{Immunohistochemistry}

Our group has extensively described immunohistochemistry of THR $\alpha$, THR $\alpha 1$ and THR $\alpha 2$ on FFPE sections (Ditsch et al. 2012a, 2013). In brief, rabbit polyclonal antibodies detecting THR $\alpha$ (Abcam, Cambridge, UK); Zytomed, Berlin, Germany), THR $\alpha 1$ (Zytomed) and THR $\alpha 2$ (Zytomed)) were stained by employing commercially available kits (Vectastain Elite rabbit-IgG-Kit (VectorLabs, Burlingame, CA); ZytoChem Plus HRP Polymer System (Zytomed). Reference sources for the used antibodies are listed in the Supplementary Table. Appropriate positive (struma, colon and placental tissue) and negative controls were included in each experiment (Supplementary Figure). Tissue sections treated with pre-immune IgGs (supersensitive rabbit negative control, BioGenex, Fremont, CA) instead of the primary antibody served as negative controls. Immunoreactivity was quantified by applying a well-established semi-quantitative scoring system (IR-score; also known as Remmele's score) by two independent observers (gynecologic pathologists (E.S. and D.M.)) by consensus. This scoring method has already been used in numerous studies (Ditsch et al. 2012b, c; Lenhard et al. 2011, 2012b) of our group. The IRS quantifies immunoreactivity by multiplication of optical staining intensity (graded as 0: no, 1: weak, 2: moderate and 3: strong staining) and the percentage of positive stained cells ( $0:$ no staining, $1: \leq 10 \%$ of the cells, $2: 11-50 \%$ of the cells, 3 : $51-80 \%$ of the cells and $4: \geq 81 \%$ of the cells). According to the previously published data, tissue samples that had been assigned an IRS higher than 1 were scored as positive (Lenhard et al. 2012a).

\section{Statistical analysis methods}

The IBM statistic package SPSS (version 25) was used to test data for statistical significance. Differences in THR expression among three or more groups were tested using the non-parametric Kruskal-Wallis rank-sum test and for pairwise comparisons using the nonparametric Mann-Whitney U rank-sum test. Correlation analysis was performed using the Spearman correlation coefficient. Overall survival (years) was compared by Kaplan-Meier graphics and differences in patient overall survival times were tested for significance using the chi-square statistics of the log rank test. For multivariate analyses, the cox regression model for overall survival was used. Data were assumed to be statistically different in case of $p<0.05$.

\section{Results}

\section{THRa expression according to EOC subtypes}

THR $\alpha$ expression showed significant differences within the histological subtype, accounting nuclear as well as cytoplasmic staining. Serous carcinomas showed only faint expression of THR $\alpha$ in the nucleus (median IRS $=2$ ) as well as in the cytoplasm (median IRS $=0$; Fig. $1 \mathrm{a}=10 \times$ lens, Fig. $1 \mathrm{f}=25 \times$ lens). A more intense staining was observed in the clear cell cases in the nucleus (median IRS $=2$ ) as well
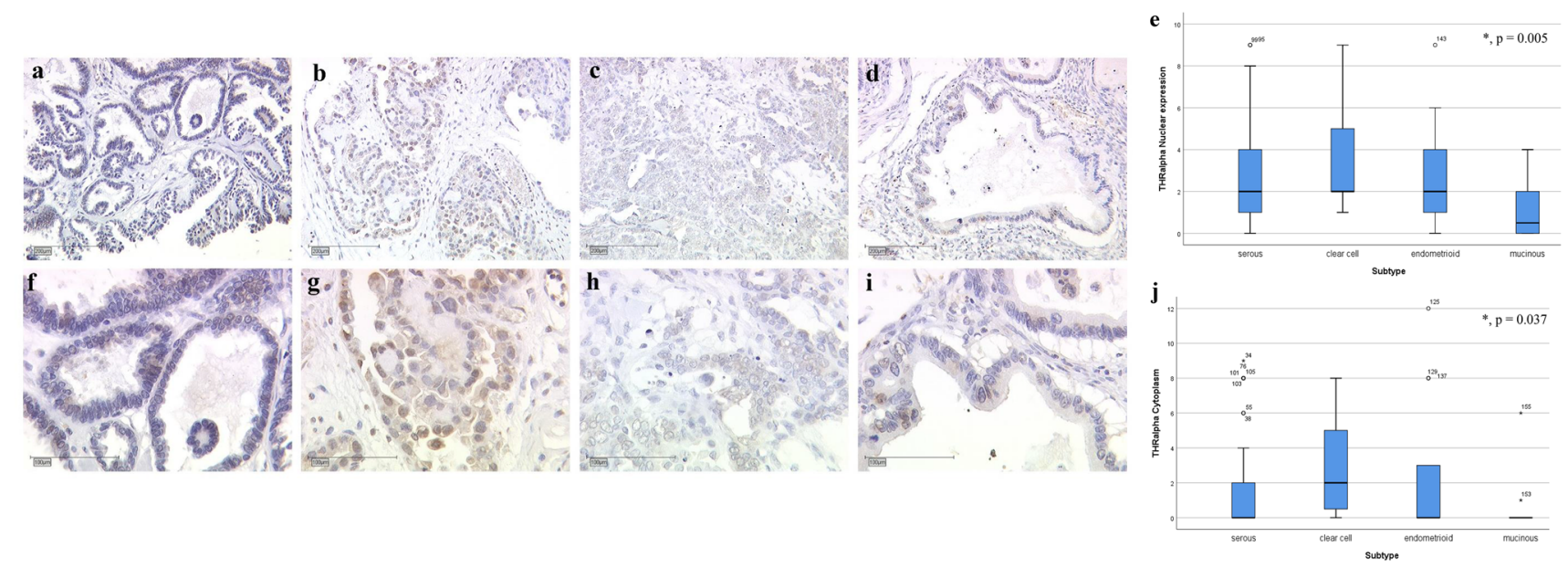

Fig. 1 a THR $\alpha$ expression in serous carcinoma $(10 \times$ lens $)$. b THR $\alpha$ expression in clear cell carcinoma $(10 \times$ lens $)$. c THR $\alpha$ expression in endometrioid carcinoma $(10 \times$ lens $)$. d THR $\alpha$ expression in mucinous carcinoma $(10 \times$ lens $)$. e Summary of THR $\alpha$ expression in different carcinoma subtypes (nuclear expression). f THR $\alpha$ expression in serous carcinoma $(25 \times$ lens $)$. $\mathbf{g}$ THR $\alpha$ expression in clear cell carcinoma $(25 \times$ lens $)$. h THR $\alpha$ expression in endometrioid carcinoma $(25 \times$ lens $)$. $\mathbf{i}$ THR $\alpha$ expression in mucinous carcinoma $(25 \times$ lens $)$. $\mathbf{j}$ Summary of THR $\alpha$ expression in different carcinoma subtypes (cytoplasmic expression) 
as in the cytoplasm (median IRS $=2$; Fig. $1 b=10 \times$ lens, Fig. $1 \mathrm{~g}=25 \times$ lens). The endometrioid subtype showed similar expression schemas as the serous subtype in the nucleus (median IRS $=2$ ) as well as in the cytoplasm (median IRS $=0$; Fig. $1 \mathrm{c}=10 \times$ lens, Fig. $1 \mathrm{~h}=25 \times$ lens). The lowest expression of THR $\alpha$ was found in the mucinous subtype in the nucleus (median IRS =1) as well as in the cytoplasm (median IRS $=0$; Fig. $1 \mathrm{~d}=10 \times$ lens, Fig. $1 \mathrm{i}=25 \times$ lens). A summary of the staining results is shown in Fig. 1e for the nuclear staining $(p=0.005)$ and Fig. $1 \mathrm{j}$ for the cytoplasmic staining $(p=0.037)$.

THR $\alpha 1$ as well as THR $\alpha 2$ showed no significant different expression according to the histological subtype. The median expression of THR $\alpha 1$ in the nucleus was 2 and the median expression in the cytoplasm was 0 . The median expression of THR $\alpha 2$ in the nucleus was 6 and, therefore, much more intense compared to THR $\alpha$ and $-\alpha 1$, respectively. The median expression of THR $\alpha 2$ in the cytoplasm was 0 . There was no significant different expression of the three THR $\alpha$ subtypes according to grading, FIGO staging or age at surgery.

\section{Correlation analyses}

Using recently published data by our institute, we were able to correlate the expression of all THR $\alpha$ subtypes stained with former investigation results. There are significant correlations with the gonadotropin receptors (Lenhard et al. 2011) and the luteinizing hormone (LH)-receptor ligand hCG (Lenhard et al. 2012b); specifically, THR $\alpha$ staining in the nucleus showed a positive correlation to the follicle stimulating hormone receptor (FSHR) (correlation coefficient $(\mathrm{cc})=0.181 ; p=0.027)$ and a negative correlation to hCG ( $c c=-0.247, p=0.003)$. In opposite, THR $\alpha$ in the cytoplasm showed a positive correlation with the luteinizing hormone/choriogonadotropin receptor (LH/hCGR) ( $\mathrm{cc}=0.199, p=0.014)$ and a positive correlation to hCG $(\mathrm{cc}=0.187, p=0.027)$. The THR $\alpha 1$ expression in the cytoplasm is positively correlated with hCG $(\mathrm{cc}=0.278$, $p=0.001)$. THR $\alpha 2$ in the nucleus showed a positive correlation to FSHR ( $\mathrm{cc}=0.185, p=0.024)$. In addition, there are also positive correlations with the classical steroid hormone receptors, which were analyzed by our research group too (Lenhard et al. 2012a). THR $\alpha$ staining in the nucleus showed a positive correlation with the $\operatorname{ER} \beta(\mathrm{cc}=0.213, p=0.009)$ and with the PR $\alpha(\mathrm{cc}=0.172, p=0.035)$. The THR $\alpha 1$ expression in the cytoplasm is positively correlated with $\operatorname{ER} \beta(\mathrm{cc}=0.219, p=0.006)$. THR $\alpha 2$ in the nucleus showed positive correlation with $\mathrm{ER} \alpha(\mathrm{cc}=0.247, p=0.002)$ and with $\mathrm{PR} \alpha(\mathrm{cc}=0.219, p=0.007)$. In addition to the classical estrogen receptors, also the GPER (Heublein et al. 2014; Heublein et al. 2013a, b) showed positive correlation with
THR $\alpha$ staining in the nucleus $(\mathrm{cc}=0.219, p=0.007)$ and with THR $\alpha 2$ in the nucleus ( $c c=0.252, p=0.002$ ).

Another positive correlation was found within the tumorassociated mucin 1 epitop (TA-MUC1) detected with the Gatipotuzumab antibody formerly known as PankoMab (Dian et al. 2013; Jeschke et al. 2012) and THR $\alpha$ staining in the nucleus ( $\mathrm{cc}=0.279, p=0.001)$. In contrast, THR $\alpha 1$ expression in the cytoplasm is negatively correlated with TA-MUC1 ( $\mathrm{cc}=-0.195, p=0.019)$. TA-MUC1 as membrane bound protein can also be translocated to the cytoplasm of tumor cells (Heublein et al. 2015). In that case, it is negatively correlated with the expression of THR $\alpha 1$ in the nucleus ( $\mathrm{cc}=-0.166, p=0.048)$ and THR $\alpha 2$ in the nucleus ( $\mathrm{cc}=-0.268, p=0.001$ ). An immunosuppressive glycoprotein that is connected to TA-MUC1 is glycodelin and its specific glycoform glycodelin A (Lenhard et al. 2013; Scholz et al. 2012). Glycodelin A showed a positive correlation with THR $\alpha 2$ in the cytoplasm ( $c c=0.170, p=0.037)$. Glycodelin showed positive correlation with THR $\alpha$ in the nucleus ( $\mathrm{cc}=0.241, p=0.003$ ) as well as in the cytoplasm $(\mathrm{cc}=0.231, p=0.004)$. THR $\alpha 2$ expression in the nucleus is positively correlated with glycodelin $(\mathrm{cc}=0.265, p=0.001)$.

\section{Survival analyses}

The expression of the general THR $\alpha$ is connected to significantly reduced overall survival in the subgroup of clear cell carcinomas. The median survival for THR $\alpha$-negative patients is 5.24 years in contrast to only 0.29 years for patients showing THR $\alpha$ expression in the nucleus (Fig. 2a, $p=0.006$ ).

The THR $\alpha$ isoforms $-\alpha 1$ and $-\alpha 2$ are in general positive prognosticators if expressed in the nucleus and negative prognosticator if expressed in the cytoplasm, respectively. In detail, THR $\alpha 1$ is a general positive prognosticator if expressed in the nucleus with a median survival of 4.22 years for patients positive for THR $\alpha 1$ and 2.08 years for patients that do not express THR $\alpha 1$ in the nucleus (Fig. $2 b, p=0.024$ ). Subgroup analyses of mucinous carcinomas showed that THR $\alpha 1$ is a negative prognosticator if expressed in the cytoplasm. The median survival time is 16.59 years for mucinous carcinoma patients that do not express THR $\alpha 1$ in the cytoplasm and 2.87 years for mucinous carcinoma patients with cytoplasmic THR $\alpha 1$ expression (Fig. 2c, $p=0.037$ ).

The THR $\alpha 2$ receptor in general is a negative prognosticator if expressed in the cytoplasm. The median survival time is 3.75 years for patients and 1.37 years for patients with THR $\alpha 2$ in the cytoplasm (Fig. 2d, $p=0.001$ ). Nuclear expression of THR $\alpha 2$ is not a general positive prognosticator. This can be found in the subgroup of serous highgrade carcinomas. The mean survival time for high-grade serous carcinoma patients with nuclear THR $\alpha 2$ expression 

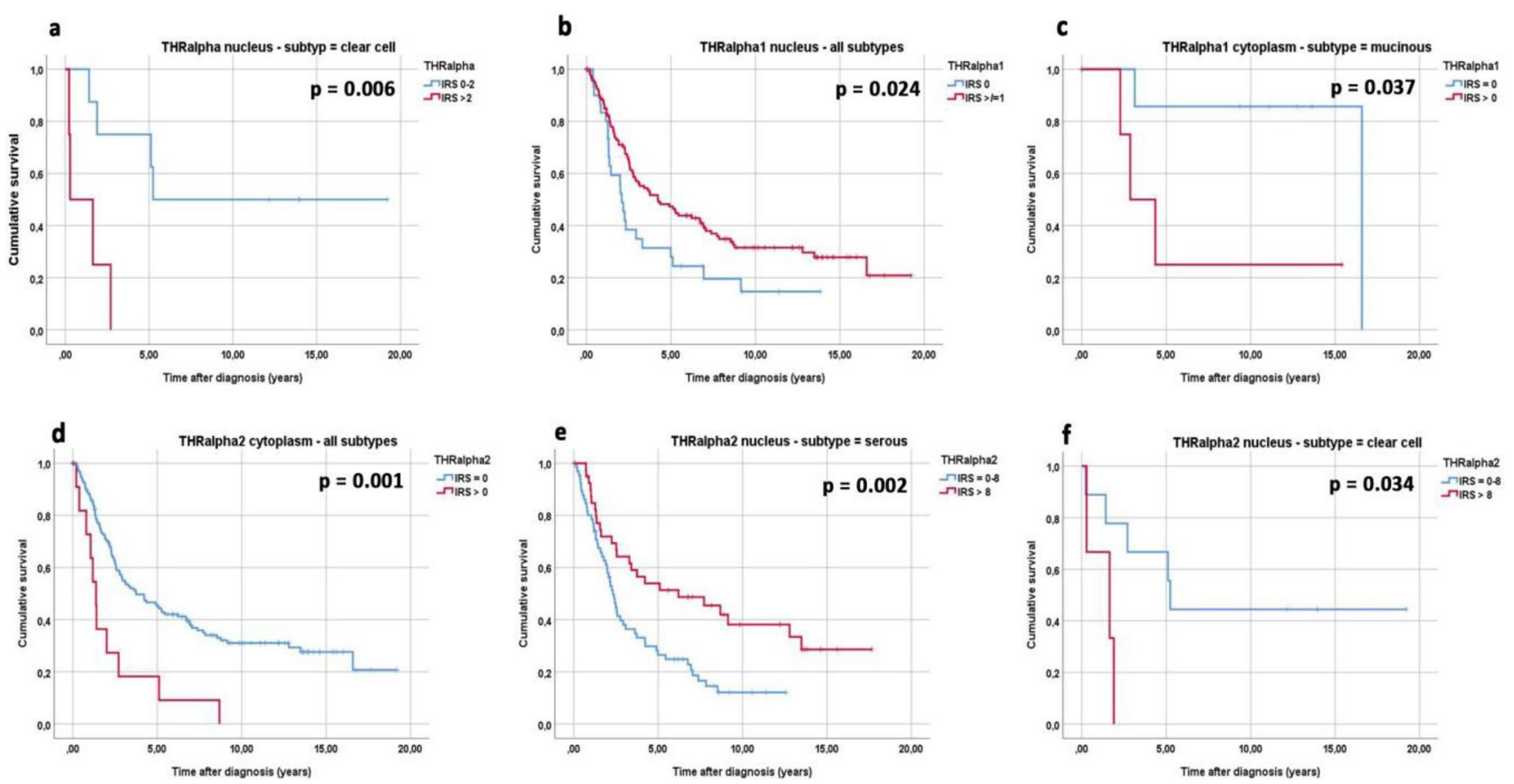

Fig. 2 Kaplan-Meier estimates of THR $\alpha$ expression, THR $\alpha 1$ expression and THR $\alpha 2$ expression were analyzed. In the clear cell subtype, patients with a high nuclear expression of THR $\alpha$ showed a significantly reduced overall survival compared with patients with a low nuclear expression (a). In addition, high nuclear THR $\alpha 1$ expression was associated with significantly better overall survival in all ovarian cancer subtypes compared to patients with a low nuclear THR $\alpha 1$ expression (b). Patients with high THR $\alpha 1$ expression in the cytoplasm and mucinous subtype had a significantly decreased overall survival compared with those mucinous carcinoma patients with low cytoplasmic expression (c). High cytoplasmic THR $\alpha 2$ expression was associated with a significantly reduced overall survival in all ovarian cancer subtypes compared to patients with a low cytoplasmic THR $\alpha 2$ expression (d). In the serous subtype, patients with a high nuclear expression of THR $\alpha 2$ showed a significantly better overall survival compared with patients with a low nuclear expression (e). Finally, in the clear cell subtype, patients with a high nuclear expression of THR $\alpha$ showed a significantly reduced very low overall survival (all patients deceased within two years) compared to patients with a low nuclear expression (f) is 6.21 years in contrast to 2.32 years for patients with no nuclear THR $\alpha 2$ expression (Fig. 2e, $p=0.002$ ). It is remarkable that patients with clear cell carcinomas show opposite results. The median survival time for clear cell carcinoma patients with nuclear THR $\alpha 2$ expression is only 1.65 years in contrast to 5.24 years for patients with no nuclear THR $\alpha 2$ expression (Fig. 2f, $p=0.034$ ).

The results of the survival analyses in correlation with the histological subtype and staining localization of THR $\alpha$, THR $\alpha 1$ and THR $\alpha 2$ are summarized in Table 2.
Table 2 Results of the survival analyses in correlation to the histological subtype and staining localization of THR $\alpha$, THR $\alpha 1$ and THR $\alpha 2$

\begin{tabular}{|c|c|c|c|c|c|}
\hline \multirow[t]{2}{*}{ Histological subtype } & \multirow{2}{*}{$\begin{array}{l}\text { THR } \alpha \\
\text { IRS }>0 \\
\text { Nucleus }\end{array}$} & \multicolumn{2}{|l|}{$\begin{array}{l}\text { THR } \alpha 1 \\
\text { IRS }>0\end{array}$} & \multicolumn{2}{|l|}{$\begin{array}{l}\text { THR } \alpha 2 \\
\text { IRS }>0\end{array}$} \\
\hline & & Nucleus & Cytoplasm & Nucleus & Cytoplasm \\
\hline Total $(n=156)$ & n.s & pos. pro. $p=0.024$ & n.s & n.s & $\begin{array}{l}\text { neg. pro } \\
p=0.001\end{array}$ \\
\hline High-grade serous $(n=84)$ & n.s & n.s & n.s & $\begin{array}{l}\text { pos. pro } \\
p=0.002\end{array}$ & n.s \\
\hline Low-grade serous $(n=26)$ & n.s & n.s & n.s & n.s & n.s \\
\hline Endometrioid $(n=21)$ & n.s & n.s & n.s & n.s & n.s \\
\hline Mucinous $(n=13)$ & n.s & n.s & $\begin{array}{l}\text { neg. pro } \\
p=0.037\end{array}$ & n.s & n.s \\
\hline Clear cell $(n=12)$ & $\begin{array}{l}\text { neg. pro } \\
p=0.006\end{array}$ & n.s & n.s & $\begin{array}{l}\text { neg. pro } \\
p=0.034\end{array}$ & n.s \\
\hline
\end{tabular}

n.s. not significant; pos. pro. positive prognosticator; neg. pro. negative prognosticator 


\section{Comparison of THRa, - $\mathbf{a} 1$ and -2 expression in low-grade and high-grade serous ovarian cancer}

As shown in Fig. 3, the expression of all three $\alpha$-subunits is higher in the nucleus of low-grade serous ovarian cancer cases with a trend to significance in the general THR $\alpha$ $(p=0.078)$, no significance for THR $\alpha 1$ and a significantly higher THR $\alpha 2$ expression in low-grade serous cancer cases compared to high-grade subtype (the receiver operating characteristic curve (ROC) analyses were performed to calculate the optimal cut-off values between low and high expression of the different THRs).

\section{Cox regression analyses of survival}

Cox regression was performed to identify independent predictors for OS. Pattern of age at surgery failed to remain significant within multivariate testing; while grading, FIGO staging, THR $\alpha 1$ in the nucleus (Table $3 \mathrm{~A}, p=0.043$ ) and THR $\alpha 2$ in the cytoplasm (Table $3 \mathrm{~B}, p=0.002$ ) were still predictive in multivariate testing sets regarding all subtypes of the study group. Due to missing clinical data in single cases, cox regression analyses were available in 146 out of 156 cases.

\section{Discussion}

Within this study, we analysed the prognostic value of the thyroid hormone receptor alpha forms 1 and 2. The general THR $\alpha$ has prognostic value only in clear cell carcinomas, where it is expressed at the highest immune scores. The differential analyses of nuclear versus cytoplasmic expression of THR $\alpha 1$ and THR $\alpha 2$ revealed striking differences concerning the overall survival of ovarian cancer patients. The thyroid hormone receptor alpha (THR $\alpha$ ) exhibits a dual role as an activator or repressor of gene transcription. Former studies showed that THR $\alpha$, formerly thought to reside solely in the nucleus and tightly bound to the DNA, shuttles rapidly between the nucleus and the cytoplasm (Bunn et al. 2001; Maruvada et al. 2003).

The role of thyroid hormones and its receptors was not very well understood in ovarian cancer biology for a longer time, only very recent publication showed their tremendous roles for this deadly disease.

Early investigations with ovarian cancer cell lines and T3, T4 and reversed T3 stimulation did not result in sufficient stimulation or inhibition outcomes (Martinez et al. 2000). Later, it was found that messenger RNA transcripts for THR $\alpha 1$, THR $\alpha 2$, T3 activating deiodinase 2 and inactivating deiodinase 3 are present in primary ovarian surface epithelial cell cultures (Rae et al. 2007). A more recent study described that for ovarian cancer patients, conflicting results were observed for $\mathrm{T} 3$ and $\mathrm{T} 4$ levels in the serum. Insignificant differences were found for T3 $(p=0.209)$ and T4 $(p=0.050)$ as compared to controls (Rasool et al. 2014).

An actual study described that $\alpha v \beta 3$ integrin, a plasma membrane receptor that binds the thyroid hormones $\mathrm{T} 3$ and $\mathrm{T} 4$, is overexpressed in ovarian cancer (Shinderman-Maman et al. 2016). Both hormones induced cell proliferation and significantly reduced the expression of genes that inhibit cell cycle particularly in ovarian cancer cells (OVCAR-3) with high integrin expression (Shinderman-Maman et al. 2016). The same group studied the expression of fifteen genes involved in DNA repair, cell cycle, apoptosis, and tumor suppression in OVCAR-3 and A2780 cell lines, using real-time PCR following short incubation with T3 or T4 (ShindermanMaman et al. 2018). The thyroid hormones downregulated the expression of the majority of genes examined, showing that these hormones influence the expression of cancerrelevant genes in ovarian cancer (Shinderman-Maman et al. 2018). The same group hypothesized that natural thyroid hormone derivatives may antagonize these actions. The three antagonists, tetraiodoacetic acid (tetrac), triiodothyroacetic
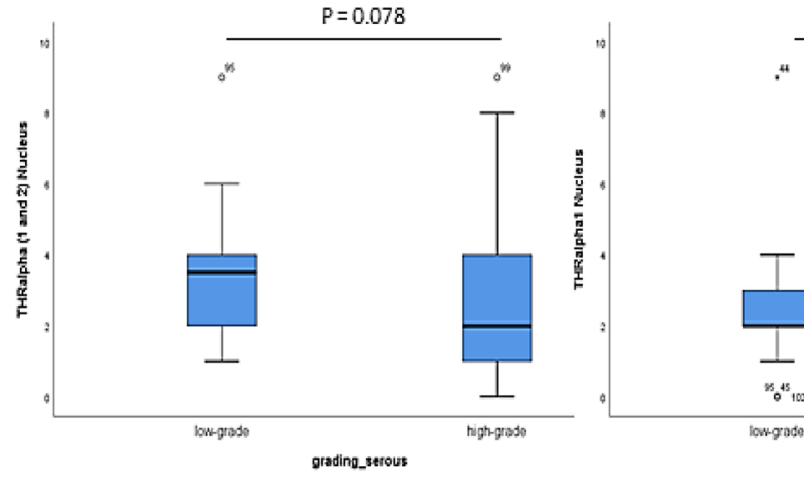

Fig. 3 Comparison of immunohistochemical staining results of the different THR (median values) in the nucleus of the high- and lowgrade serous ovarian cancer subtypes. (IRS Immunoreactive Score,
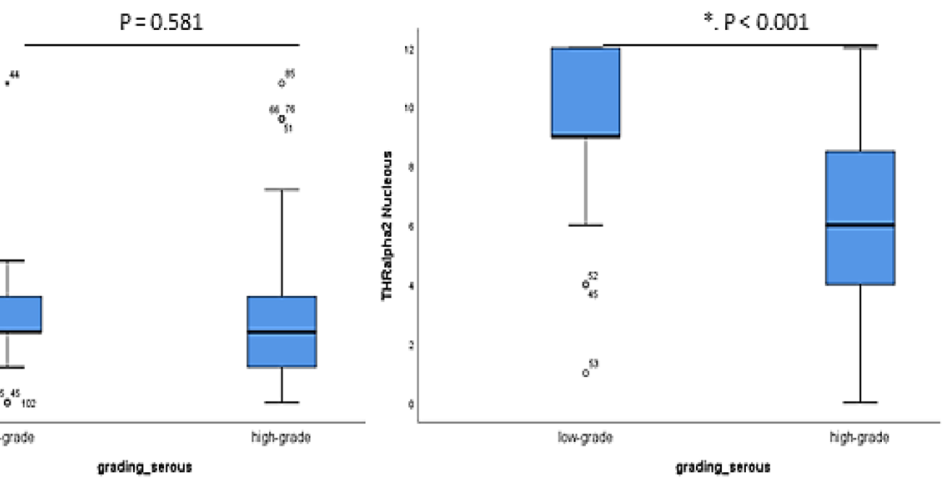

THR: Thyroid Receptor). The expression of THR $\alpha 2$ in the nucleus is significantly different in low-grade compared to high-grade serous carcinomas (marked by an asterisk) 
Table 3 Multivariate survival analyses with the overall survival time for (A) THR $\alpha 1$ expression in the nucleus, (B) THR $\alpha 2$ expression in the cytoplasm, regarding patients age, histological subtype, grading, and staging $(n=146)$

\begin{tabular}{|c|c|c|c|c|}
\hline \multirow[t]{2}{*}{ Variables } & \multirow[t]{2}{*}{$p$ value } & \multirow[t]{2}{*}{ Hazard ratio } & \multicolumn{2}{|c|}{$\begin{array}{l}95.0 \% \text { Confidence } \\
\text { Interval }\end{array}$} \\
\hline & & & Lower & Upper \\
\hline \multicolumn{5}{|l|}{ (A) THR $\alpha 1$ (nucleus) } \\
\hline IRS $>0$ versus IRS 0 & 0.049 & 0.618 & 0.383 & 0.997 \\
\hline \multicolumn{5}{|l|}{ FIGO } \\
\hline I/II versus III/IV & 0.001 & 2.761 & 1.510 & \\
\hline \multicolumn{5}{|l|}{ Grading } \\
\hline G1/low grade versus & 0.002 & 2.753 & 1.457 & 5.199 \\
\hline \multicolumn{5}{|l|}{ G2/3/high grade } \\
\hline \multicolumn{5}{|l|}{ Histological subtype } \\
\hline all subtypes versus high-grade serous & 0.964 & 0.994 & 0.783 & 1.263 \\
\hline \multicolumn{5}{|l|}{ Age } \\
\hline$<60$ versus $\geq 60$ years & 0.116 & 0.717 & 0.473 & 1.085 \\
\hline \multicolumn{5}{|l|}{ (B) THR $\alpha 2$ (cytoplasm) } \\
\hline IRS $>0$ versus IRS 0 & 0.002 & 2.790 & 1.466 & 5.310 \\
\hline \multicolumn{5}{|l|}{ Age } \\
\hline$<60$ versus $\geq 60$ years & 0.212 & 0.769 & 0.509 & 1.161 \\
\hline \multicolumn{5}{|l|}{ Histological subtype } \\
\hline all other subtypes versus high-grade serous & 0.673 & 0.950 & 0.747 & 1.207 \\
\hline \multicolumn{5}{|l|}{ Grading } \\
\hline G1/low-grade versus & 0.001 & 0.325 & 0.171 & 0.618 \\
\hline \multicolumn{5}{|l|}{ G2/3/high-grade } \\
\hline \multicolumn{5}{|l|}{ FIGO } \\
\hline $\mathrm{I} / \mathrm{II}$ versus III/IV & 0.001 & 0.365 & 0.201 & 0.662 \\
\hline
\end{tabular}

acid (triac) and 3-iodothyronamine (T1AM) inhibited cell proliferation and induced cell death and DNA damage in the two ovarian cancer cell lines (OVCAR3 and A2780). Therefore, they concluded that the cytotoxic potential of thyroid hormone derivatives, tetrac, triac and T1AM, in ovarian cancer might provide a much-needed novel therapeutic approach (Shinderman-Maman et al. 2017).

Based on the results of the former study, another group described that thyroid hormone causes elevated phosphorylation and nuclear enrichment of $\mathrm{ER} \alpha$ (Hsieh et al. 2017). In addition, confocal microscopy indicated that both T4 and estradiol caused nuclear translocation of integrin $\alpha \mathrm{v}$ and phosphorylation of ER $\alpha$ (Hsieh et al. 2017). Within our study, we found a positive correlation between the THR $\alpha 2$ in the nucleus and $\mathrm{ER} \alpha$. We also found positive correlation of THR $\alpha$ in the nucleus and ER $\beta$, assuming that thyroid hormones not only elevate the nuclear enrichment of ER $\alpha$ but also might influence ER $\beta$. However, our correlations referred to the whole study cohort and did not focus on the histological subtypes. Another study showed that THR $\alpha 1$ inhibits the $\mathrm{ER} \alpha$ transactivation from the consensus estrogen response element (ERE). In contrast, the ligand bound THR $\beta 1$ facilitates ER $\beta$-mediated transactivation (Vasudevan et al. 2001). We also found a positive correlation between the GPER and THR $\alpha$. Sheng et al. showed that the GPER together with integrin $\alpha v \beta 3$ participate in the induction of male germ cell proliferation and thyroid transcription disruption after low-dose Bisphenol A treatment (Sheng et al. 2019). Another correlation of our study was found between THR $\alpha$ in the nucleus and the FSH receptor; whereas, the THR $\alpha$ expression in the cytoplasm showed a positive correlation to the $\mathrm{LH} / \mathrm{hCG}$ receptor. It has been known for a longer time that LH, FSH, and TSH show low-level crossreactivity between their respective receptors (Tonacchera et al. 2006). Vissenberg et al. explained that T3 in combination with FSH enhances granulosa cell proliferation and inhibits granulosa cell apoptosis by the PI3K/Akt pathway (Vissenberg et al. 2015). They also described that T3 is considered a biological amplifier of the stimulatory action of gonadotrophins on granulosa cell function (Vissenberg et al. 2015). Because the exclusive expression of the FSHR has already been described by our group as a negative prognosticator in ovarian cancer cases, our finding about enhanced expression of both FSHR and THR $\alpha$ in the nucleus might lead to new treatment strategies for this type of cancer (Lenhard et al. 2011). This assumption might also apply for the antibody Gatipotuzumab and its TA-MUC1 epitope (Heublein et al. 2019), which showed an inverse correlation to THR $\alpha 1$ and -2 expression either in the nucleus or in the cytoplasm, respectively. 
In addition, T4 has been shown to promote ovarian cancer cell proliferation via integrin $\alpha v \beta 3$. T4 also induced the activation of ERK1/2 and expression of programmed death-ligand 1 (PD-L1) in ovarian cancer cells (Chin et al. 2018). In contrast, resveratrol binds to integrin $\alpha v \beta 3$ at a discrete site and induces p53-dependent anti-proliferation in malignant neoplastic cells. T4 impairs resveratrolinduced anti-proliferation in human ovarian cancer cells and T4 inhibited resveratrol-induced nuclear accumulation of COX-2 (Chin et al. 2018). Furthermore, T4 increased expression and cytoplasmic accumulation of PD-L1, which in turn acted to retain inducible COX-2 in the cytoplasm (Chin et al. 2018). Thus, T4 inhibits COX-2-dependent apoptosis in ovarian cancer cells by retaining inducible COX-2 with PD-L1 in the cytoplasm (Chin et al. 2018).

Recently, the interplay between epithelial-mesenchymal transition (EMT) and the thyroid hormones- $\alpha v \beta 3$ axis in ovarian cancer was investigated (Weingarten et al. 2018). It was shown that the transcription of mesenchymal markers, $\beta$-catenin, zeb-1, slug/snail, vimentin, and n-cadherin was hardly affected by $\mathrm{T} 3$ and T4, while that of the epithelial markers, e-cadherin and zo-1, and was inhibited after treatment with thyroid hormones. These results suggest a novel role for the thyroid hormone- $\alpha v \beta 3$ axis in EMT, with possible implications for ovarian cancer metastasis (Weingarten et al. 2018).

Finally, a group investigated the role of the thyroid hormone receptor Interactor 13 (TRIP13) in epithelial ovarian cancer (EOC) (Zhou and Shu, 2019). Bioinformatics analysis showed that TRIP13 was one of the most significantly upregulated proteins in EOC. Results of the described study showed that TRIP13 acted as an oncopromotive regulator in EOC development by modulating the Notch signaling pathway (Zhou and Shu, 2019).

A large demographic study, the "Ovarian Cancer Association Consortium", showed that hyperthyroidism within the 5 years before ovarian cancer diagnosis was associated with an increased risk of death (Minlikeeva et al. 2017). These very recent results were accompanied by the fact that a more modest association was observed with the history of hypothyroidism ( $n=624$ cases) and mortality (Minlikeeva et al. 2017).

In sum, the results of the experimental and demographic studies about the roles of thyroid hormones, its receptors and interacting proteins. There is growing body of evidence that they play a major role in ovarian cancer biology and survival of ovarian cancer patients. Only recent studies were able to bring new light into this area of research.

\section{Conclusions}

With our study, we could show that there is a direct link between nuclear expression of THR $\alpha 1$ or -2 and better survival in EOC, except for the subgroup of clear cell carcinomas. The latter group seems to have different properties concerning THR $\alpha$ expression. Shifting the expression of THR $\alpha 1$ or -2 to the cytoplasm seems to be connected with reduced overall survival in EOC cases. Therefore, the search for THR $\alpha$ interacting factors that prevent this shift to the cytoplasm seems to be a useful new approach for the search of future treatment strategies against the threatening disease of Epithelial Ovarian Cancer.

Acknowledgments Open Access funding provided by Projekt DEAL. We would like to thank S. Kunze ${ }^{\dagger}$ for technical assistance. Further, we would like to thank V. Lanser for their help with retrieving follow-up data.

Author contributions ND, SH, UJ, DM conceived and designed the experiments. CS, CK, AH, ES performed the experiments. BS, FT, $\mathrm{SM}$, JE analyzed the data. ND, UJ wrote the paper. All authors have read and approved the manuscript.

\section{Compliance with ethical standards}

Conflict of interest Sabine Heublein reports grants from FERRING, personal fees from Roche, other from Astra Zeneca, grants from Novartis Oncology, grants and non-financial support from Apceth $\mathrm{GmbH}$, non-financial support from Addex. Fabian Trillsch declares Research support, advisory board, honoraria and travel expenses from AstraZeneca, Clovis, Medac, PharmaMar, Roche, Tesaro. Sven Mahner reports grants and personal fees from AstraZeneca, personal fees from Clovis, grants and personal fees from Medac, grants and personal fees from MSD. He also reports personal fees from Novartis, grants and personal fees from PharmaMar, grants and personal fees from Roche, personal fees from Sensor Kinesis, grants and personal fees from Tesaro, grants and personal fees from Teva, outside the submitted work. The remaining authors declare no conflict of interest.

Ethics approval and consent to participate The Ethics Committee of the Ludwig-Maximilians-University, Munich, Germany (approval number 227-09) on 30 September 2009, approved the study. All tissue samples used for this study were obtained from leftover material from the archives of LMU Munich, Department Gynaecology and Obstetrics, Ludwig-Maximilians-University, Munich, Germany, initially used for pathological diagnostics. When this retrospective study was initiated, diagnostic procedures had already been fully completed; the tissue samples were classified as leftover material and underwent irreversible anonymization. Under these circumstances, no individual written informed consent was needed as per declaration of the Ethics Committee of the Ludwig-Maximilians-University. All experiments were performed according to the standards of the Declaration of Helsinki (1975).

Open Access This article is licensed under a Creative Commons Attribution 4.0 International License, which permits use, sharing, adaptation, distribution and reproduction in any medium or format, as long as you give appropriate credit to the original author(s) and the source, provide a link to the Creative Commons licence, and indicate if changes were made. The images or other third party material in this article are included in the article's Creative Commons licence, unless indicated otherwise in a credit line to the material. If material is not included in the article's Creative Commons licence and your intended use is not permitted by statutory regulation or exceeds the permitted use, you will need to obtain permission directly from the copyright holder. To view a copy of this licence, visit http://creativecommons.org/licenses/by/4.0/. 


\section{References}

Beachy PA, Karhadkar SS, Berman DM (2004) Mending and malignancy. Nature 431:402

Bunn CF, Neidig JA, Freidinger KE, Stankiewicz TA, Weaver BS, McGrew J, Allison LA (2001) Nucleocytoplasmic shuttling of the thyroid hormone receptor alpha. Mol Endocrinol 15:512-533

Chin YT, Wei PL, Ho Y, Nana AW, Changou CA, Chen YR, Yang YS, Hsieh MT, Hercbergs A, Davis PJ, Shih YJ, Lin HY (2018) Thyroxine inhibits resveratrol-caused apoptosis by PD-L1 in ovarian cancer cells. Endocr Relat Cancer 25:533-545

Cohen K, Flint N, Shalev S, Erez D, Baharal T, Davis PJ, Hercbergs A, Ellis M, Ashur-Fabian O (2014) Thyroid hormone regulates adhesion, migration and matrix metalloproteinase 9 activity via alphavbeta3 integrin in myeloma cells. Oncotarget 5:6312-6322

Dian D, Lenhard M, Mayr D, Heublein S, Karsten U, Goletz S, Kuhn C, Wiest I, Friese K, Weissenbacher T, Jeschke U (2013) Staining of MUC1 in ovarian cancer tissues with PankoMab-GEX detecting the tumour-associated epitope, TA-MUC1, as compared to antibodies HMFG-1 and 115D8. Histol Histopathol 28:239-244

Ditsch N, Mayr D, Lenhard M, Strauss C, Vodermaier A, Gallwas J, Stoeckl D, Graeser M, Weissenbacher T, Friese K, Jeschke U (2012a) Correlation of thyroid hormone, retinoid X, peroxisome proliferator-activated, vitamin D and oestrogen/progesterone receptors in breast carcinoma. Oncol Lett 4:665-671

Ditsch N, Toth B, Himsl I, Lenhard M, Ochsenkuhn R, Friese K, Mayr D, Jeschke U (2013) Thyroid hormone receptor (TR)alpha and TRbeta expression in breast cancer. Histol Histopathol 28:227-237

Ditsch N, Toth B, Mayr D, Lenhard M, Gallwas J, Weissenbacher T, Dannecker C, Friese K, Jeschke U (2012b) The association between vitamin $\mathrm{D}$ receptor expression and prolonged overall survival in breast cancer. J Histochem Cytochem 60:121-129

Ditsch N, Vrekoussis T, Lenhard M, Ruhl I, Gallwas J, Weissenbacher T, Friese K, Mayr D, Makrigiannakis A, Jeschke U (2012c) Retinoid $\mathrm{X}$ receptor alpha (RXRalpha) and peroxisome proliferatoractivated receptor gamma (PPARgamma) expression in breast cancer: an immunohistochemical study. Vivo 26:87-92

Espey LL (1994) Current status of the hypothesis that mammalian ovulation is comparable to an inflammatory reaction. Biol Reprod 50:233-238

Heublein S, Mayr D, Egger M, Karsten U, Goletz S, Angele M, Gallwas J, Jeschke U, Ditsch N (2015) Immunoreactivity of the fully humanized therapeutic antibody PankoMab-GEX is an independent prognostic marker for breast cancer patients. J Exp Clin Cancer Res 34:50

Heublein S, Mayr D, Friese K, Jarrin-Franco MC, Lenhard M, Mayerhofer A, Jeschke U (2014) The G-protein-coupled estrogen receptor (GPER/GPR30) in ovarian granulosa cell tumors. Int J Mol Sci 15:15161-15172

Heublein S, Mayr D, Vrekoussis T, Friese K, Hofmann SS, Jeschke U, Lenhard M (2013a) The G-protein coupled estrogen receptor (GPER/GPR30) is a gonadotropin receptor dependent positive prognosticator in ovarian carcinoma patients. PLoS ONE 8:e71791

Heublein S, Page S, Mayr D, Schmoeckel E, Trillsch F, Marme F, Mahner S, Jeschke U, Vattai A (2019) Potential Interplay of the Gatipotuzumab Epitope TA-MUC1 and Estrogen Receptors in Ovarian Cancer. Int J Mol Sci 20:295

Heublein S, Vrekoussis T, Kuhn C, Friese K, Makrigiannakis A, Mayr D, Lenhard M, Jeschke U (2013b) Inducers of G-protein coupled estrogen receptor (GPER) in endometriosis: potential implications for macrophages and follicle maturation. J Reprod Immunol 97:95-103

Hsieh MT, Wang LM, Changou CA, Chin YT, Yang YSH, Lai HY, Lee SY, Yang YN, Whang-Peng J, Liu LF, Lin HY, Mousa SA, Davis
PJ (2017) Crosstalk between integrin alphavbeta3 and ERalpha contributes to thyroid hormone-induced proliferation of ovarian cancer cells. Oncotarget 8:24237-24249

Jeschke U, Wiest I, Schumacher AL, Kupka M, Rack B, Stahn R, Karsten U, Mayr D, Friese K, Dian D (2012) Determination of MUC1 in sera of ovarian cancer patients and in sera of patients with benign changes of the ovaries with CA15-3, CA27.29, and PankoMab. Anticancer Res 32:2185-2189

Lazar MA (1993) Thyroid hormone receptors: multiple forms, multiple possibilities. Endocr Rev 14:184-193

Lenhard M, Heublein S, Kunert-Keil C, Vrekoussis T, Lomba I, Ditsch N, Mayr D, Friese K, Jeschke U (2013) Immunosuppressive Glycodelin $\mathrm{A}$ is an independent marker for poor prognosis in endometrial cancer. BMC Cancer 13:616

Lenhard M, Lennerova T, Ditsch N, Kahlert S, Friese K, Mayr D, Jeschke U (2011) Opposed roles of follicle-stimulating hormone and luteinizing hormone receptors in ovarian cancer survival. Histopathology 58:990-994

Lenhard M, Tereza L, Heublein S, Ditsch N, Himsl I, Mayr D, Friese K, Jeschke U (2012a) Steroid hormone receptor expression in ovarian cancer: progesterone receptor B as prognostic marker for patient survival. BMC Cancer 12:553

Lenhard M, Tsvilina A, Schumacher L, Kupka M, Ditsch N, Mayr D, Friese K, Jeschke U (2012b) Human chorionic gonadotropin and its relation to grade, stage and patient survival in ovarian cancer. BMC Cancer 12:2

Li J, Lin Q, Yoon HG, Huang ZQ, Strahl BD, Allis CD, Wong J (2002) Involvement of histone methylation and phosphorylation in regulation of transcription by thyroid hormone receptor. Mol Cell Biol 22:5688-5697

Ling Y, Xu X, Hao J, Ling X, Du X, Liu X, Zhao X (2010) Aberrant methylation of the THRB gene in tissue and plasma of breast cancer patients. Cancer Genet Cytogenet 196:140-145

Martinez MB, Ruan M, Fitzpatrick LA (2000) Altered response to thyroid hormones by breast and ovarian cancer cells. Anticancer Res 20:4141-4146

Maruvada P, Baumann CT, Hager GL, Yen PM (2003) Dynamic shuttling and intranuclear mobility of nuclear hormone receptors. $\mathrm{J}$ Biol Chem 278:12425-12432

Minlikeeva AN, Freudenheim JL, Cannioto RA, Eng KH, Szender JB, Mayor P, Etter JL, Cramer DW, Diergaarde B, Doherty JA, Dork T, Edwards R, Defazio A, Friel G, Goodman MT, Hillemanns P, Hogdall E, Jensen A, Jordan SJ, Karlan BY, Kjaer SK, Klapdor R, Matsuo K, Mizuno M, Nagle CM, Odunsi K, Paddock L, Rossing MA, Schildkraut JM, Schmalfeldt B, Segal BH, Starbuck K, Terry KL, Webb PM, Zsiros E, Ness RB, Modugno F, Bandera EV, Chang-Claude J, Moysich KB (2017) History of thyroid disease and survival of ovarian cancer patients: results from the Ovarian Cancer Association Consortium, a brief report. Br J Cancer 117:1063-1069

Murdoch WJ (1998) Perturbation of sheep ovarian surface epithelial cells by ovulation: evidence for roles of progesterone and poly(ADP-ribose) polymerase in the restoration of DNA integrity. J Endocrinol 156:503-508

Murdoch WJ, Wilken C, Young DA (1999) Sequence of apoptosis and inflammatory necrosis within the formative ovulatory site of sheep follicles. J Reprod Fertil 117:325-329

Ness RB, Cottreau C (1999) Possible role of ovarian epithelial inflammation in ovarian cancer. J Natl Cancer Inst 91:1459-1467

Ness RB, Grisso JA, Cottreau C, Klapper J, Vergona R, Wheeler JE, Morgan M, Schlesselman JJ (2000) Factors related to inflammation of the ovarian epithelium and risk of ovarian cancer. Epidemiology 11:111-117

Rae MT, Gubbay O, Kostogiannou A, Price D, Critchley HO, Hillier SG (2007) Thyroid hormone signaling in human ovarian surface epithelial cells. J Clin Endocrinol Metab 92:322-327 
Rae MT, Hillier SG (2005) Steroid signalling in the ovarian surface epithelium. Trends Endocrinol Metab 16:327-333

Rae MT, Niven D, Ross A, Forster T, Lathe R, Critchley HO, Ghazal $P$, Hillier SG (2004) Steroid signalling in human ovarian surface epithelial cells: the response to interleukin-1alpha determined by microarray analysis. J Endocrinol 183:19-28

Rasmusson B, Feldt-Rasmussen U, Hegedus L, Perrild H, Bech K, Hoier-Madsen M (1987) Thyroid function in patients with breast cancer. Eur J Cancer Clin Oncol 23:553-556

Rasool M, Naseer MI, Zaigham K, Malik A, Riaz N, Alam R, Manan A, Sheikh IA, Asif M (2014) Comparative study of alterations in Tri-iodothyronine (T3) and Thyroxine (T4) Hormone Levels in Breast and Ovarian Cancer. Pak J Med Sci 30:1356-1360

Scholz C, Heublein S, Lenhard M, Friese K, Mayr D, Jeschke U (2012) Glycodelin A is a prognostic marker to predict poor outcome in advanced stage ovarian cancer patients. BMC Res Notes 5:551

Sheng Z, Wang C, Ren F, Liu Y, Zhu B (2019) Molecular mechanism of endocrine-disruptive effects induced by Bisphenol A: the role of transmembrane G-protein estrogen receptor 1 and integrin alphavbeta3. J Environ Sci (China) 75:1-13

Shinderman-Maman E, Cohen K, Moskovich D, Hercbergs A, Werner H, Davis PJ, Ellis M, Ashur-Fabian O (2017) Thyroid hormones derivatives reduce proliferation and induce cell death and DNA damage in ovarian cancer. Sci Rep 7:16475

Shinderman-Maman E, Cohen K, Weingarten C, Nabriski D, Twito O, Baraf L, Hercbergs A, Davis PJ, Werner H, Ellis M, Ashur-Fabian O (2016) The thyroid hormone-alphavbeta3 integrin axis in ovarian cancer: regulation of gene transcription and MAPK-dependent proliferation. Oncogene 35:1977-1987

Shinderman-Maman E, Weingarten C, Moskovich D, Werner H, Hercbergs A, Davis PJ, Ellis M, Ashur-Fabian O (2018) Molecular insights into the transcriptional regulatory role of thyroid hormones in ovarian cancer. Mol Carcinog 57:97-105

Silva JM, Dominguez G, Gonzalez-Sancho JM, Garcia JM, Silva J, Garcia-Andrade C, Navarro A, Munoz A, Bonilla F (2002)
Expression of thyroid hormone receptor/erbA genes is altered in human breast cancer. Oncogene 21:4307-4316

Tonacchera M, Ferrarini E, Dimida A, Agretti P, de Marco G, Pinchera A, Sanders J, Evans M, Richards T, Furmaniak J, Smith BR (2006) Effects of a thyroid-stimulating human monoclonal autoantibody (M22) on functional activity of LH and FSH receptors. Thyroid 16:1085-1089

Turken O, Narin Y, Demirbas S, Onde ME, Sayan O, Kandemir EG, Yaylac IM, Ozturk A (2003) Breast cancer in association with thyroid disorders. Breast Cancer Res 5:R110-R113

Vasudevan N, Koibuchi N, Chin WW, Pfaff DW (2001) Differential crosstalk between estrogen receptor (ER)alpha and ERbeta and the thyroid hormone receptor isoforms results in flexible regulation of the consensus ERE. Brain Res Mol Brain Res 95:9-17

Vissenberg R, Manders VD, Mastenbroek S, Fliers E, Afink GB, RisStalpers C, Goddijn M, Bisschop PH (2015) Pathophysiological aspects of thyroid hormone disorders/thyroid peroxidase autoantibodies and reproduction. Hum Reprod Update 21:378-387

Weingarten C, Jenudi Y, Tshuva RY, Moskovich D, Alfandari A, Hercbergs A, Davis PJ, Ellis M, Ashur-Fabian O (2018) The interplay between Epithelial-Mesenchymal Transition (EMT) and the thyroid hormones-alphavbeta3 axis in ovarian cancer. Horm Cancer 9:22-32

Zhang J, Lazar MA (2000) The mechanism of action of thyroid hormones. Annu Rev Physiol 62:439-466

Zhou XY, Shu XM (2019) TRIP13 promotes proliferation and invasion of epithelial ovarian cancer cells through Notch signaling pathway. Eur Rev Med Pharmacol Sci 23:522-529

Publisher's Note Springer Nature remains neutral with regard to jurisdictional claims in published maps and institutional affiliations.

\section{Affiliations}

\section{Nina Ditsch ${ }^{1,5} \cdot$ Sabine Heublein $^{1,2}$. Udo Jeschke ${ }^{1,5} \cdot$ Cornelia Sattler $^{1} \cdot$ Christina Kuhn $^{1}$ - Anna Hester ${ }^{1}$. Bastian Czogalla ${ }^{1} \cdot$ Fabian Trillsch $^{1} \cdot$ Sven Mahner ${ }^{1}$ - Jutta Engel ${ }^{3}$ - Doris Mayr ${ }^{4} \cdot$ Elisa Schmoeckel $^{4}$ (I)}

Nina Ditsch

nina.ditsch@med.uni-muenchen.de

Sabine Heublein

sabine.heublein@med.uni-heidelberg.de

Cornelia Sattler

connisattler@gmail.com

Christina Kuhn

christina.kuhn@med.uni-muenchen.de

Anna Hester

anna.hester@med.uni-muenchen.de

Bastian Czogalla

bastian.czogalla@med.uni-muenchen.de

Fabian Trillsch

fabian.trillsch@med.uni-muenchen.de

Sven Mahner

sven.mahner@med.uni-muenchen.de

Jutta Engel

engel@ibe.med.uni-muenchen.de
Doris Mayr

doris.mayr@med.uni-muenchen.de

Elisa Schmoeckel

elisa.schmoeckel@med.uni-muenchen.de

1 Department of Obstetrics and Gynecology, LMU Munich, University Hospital, Marchioninistr. 15, 81377 Munich, Germany

2 Department of Obstetrics and Gynecology, Heidelberg University Hospital, Ruprecht-Karls-University of Heidelberg, 69120 Heidelberg, Germany

3 Munich Cancer Registry (MCR), Bavarian Cancer RegistryRegional Centre Munich (LGL), Institute for Medical Information Processing, Biometry and Epidemiology (IBE), Ludwig-Maximilians-University (LMU), Munich, Germany

4 Department of Pathology, LMU Munich, Thalkirchner Str. 36, 80337 Munich, Germany

5 Department of Obstetrics and Gynecology, University Hospital Augsburg, Stenglinstrasse 2, 86156 Augsburg, Germany 